\title{
MS34-P01 | CRystal StRucture AND HiRShfeld sURfaCe ANALYSIS OF 1,3,4-thiadiazol
}

\section{DERIVATIVE}

TABTI, Charef (UNIVERSITY OF MOSTAGANEM, MOSTAGANEM, DZA)

1,3,4-thiadiazol derivative has been synthesized. Its structural properties were investigated using density functional theory (DFT) by employing B3LYP with 6-311G (d,p) basis set. Its crystalline structure was determined using $X$ ray diffraction data. The theoretical results from DFT are in good agreement with the $X$ ray diffraction. $A$ good agreement with the structure determined from the single crystal measurements was found. Hirshfeld surface analyses (HS) and spectral analysis using IR, RMN- ${ }^{1} \mathrm{H}$ and $\mathrm{RMN}-{ }^{13} \mathrm{C}$ were performed. The frontier molecular orbitals (HOMO and LUMO) were simulated. They showed a small energy gap which confirms that charge transfer takes place. Electrostatic potential (MEP) surface was simulated demonstrating the existence of reactive sites in the title molecule. Nonlinear optical (NLO) behavior of the examined molecule was investigated by the determination of the electric dipole moment $\mu$, the polarizability $\alpha$ and the hyperpolarizability $\beta$ using the B3LYP method. 